\section{Thrombopoietin maintains cell numbers of hematopoietic stem and progenitor cells with megakaryopoietic potential}

\author{
Aled O'Neill, ${ }^{1}$ Desmond Chin, ${ }^{1}$ Darren Tan, ${ }^{1}$ A'Qilah Banu Bte Abdul Majeed, ${ }^{1}$ \\ Ayako Nakamura-Ishizu ${ }^{1,2}$ and Toshio Suda ${ }^{1,2}$ \\ ${ }^{1}$ Cancer Science Institute, National University of Singapore, Singapore and ${ }^{2}$ International \\ Research Center for Medical Sciences, Kumamoto University, Kumamoto, Japan
}

\section{ABSTRACT}

hrombopoietin has long been known to influence megakaryopoiesis and hematopoietic stem and progenitor cells, although the exact mechanisms through which it acts are unknown. Here we show that MPL expression correlates with megakaryopoietic potential of hematopoietic stem and progenitor cells and identify a population of quiescent hematopoietic stem and progenitor cells that show limited dependence on thrombopoietin signaling. We show that thrombopoietin is primarily responsible for maintenance of hematopoietic cells with megakaryocytic differentiation potential and their subsequent megakaryocyte differentiation and maturation. The loss of megakaryocytes in thrombopoietin knockout mouse models results in a reduction of megakaryocyte-derived chemokine platelet factor 4 (CXCL4/PF4) in the bone marrow and administration of recombinant CXCL4/PF4 rescues the loss of quiescence observed in these mice. CXCL4/PF4 treatment does not rescue reduced hematopoietic stem and progenitor cell numbers, suggesting that thrombopoietin maintains hematopoietic stem and progenitor cell numbers directly.

\section{Introduction}

Hematopoietic stem cells (HSC) are defined by their ability to not only differentiate into all blood cell lineages, but also to self-renew and enter the non-proliferative quiescent state. ${ }^{1}$ Maintenance of this quiescent state is thought to rely on several spatially separated niche factors ${ }^{2}$ and there are many candidates for potential ex-vivo maintenance of HSC quiescence including CXCL12 and thrombopoietin (THPO). ${ }^{3}$ It has long been known that loss of THPO signaling results in reduced HSC numbers and loss of quiescence,,, 5 although the mechanisms behind this phenomenon are yet to be identified. In addition to influencing quiescence, THPO is known to drive megakaryocyte differentiation and maturation as well as platelet production. ${ }^{6}$

The THPO receptor, myeloproliferative leukemia (MPL), is expressed on HSC and it has been shown that HSC require THPO for survival in vitro.' Cells phenotypically similar to HSC in the hematopoietic compartment have also been shown to differentiate directly into megakaryocytes without cell division, suggesting a close relationship between the earliest megakaryocyte progenitors (MkP) and HSC. ${ }^{8}$ Determining how THPO is responsible for these seemingly different effects has been an object of much research, with several studies on mutated leukemia cell lines suggesting that the expression ratios between MPL and its downstream signal transducer JAK2 as well as extracellular THPO levels may determine whether hematopoietic stem and progenitor cells (HSPC) enter quiescent or proliferative pathways. ${ }^{9,10}$ Whether HSC in the bone marrow have different responses to THPO remains to be seen. We, therefore, set out to investigate the effects of THPO on various HSC both in vivo and in vitro.

\section{Methods}

\section{Experimental mice}

All experiments were performed on 8- to 12-week-old mice in the C57BL/6-NTac back-
Ferrata Storti Foundation
Haematologica 2021

Volume 106(7):1883-1891

\section{Correspondence: \\ TOSHIO SUDA \\ sudato@keio.jp}

Received: October 23, 2019.

Accepted: May 28, 2020.

Pre-published: June 11, 2020

https://doi.org/10.3324/haematol.2019.241406

(c)2021 Ferrata Storti Foundation

Material published in Haematologica is covered by copyright. All rights are reserved to the Ferrata Storti Foundation. Use of published material is allowed under the following terms and conditions:

https://creativecommons.org/licenses/by-nc/4.0/legalcode. Copies of published material are allowed for personal or internal use. Sharing published material for non-commercial purposes is subject to the following conditions:

https://creativecommons.org/licenses/by-nc/4.0/legalcode, sect. 3. Reproducing and sharing published material for commercial purposes is not allowed without permission in writing from the publisher. 
ground in accordance with Institutional Animal Care and Use Committee protocols. Alb-Cre mice in a white background were kindly provided by Prof. Oingde Wang of the University of Pittsburgh Medical Center and were back-crossed with C57BL/6-NTac mice at least five times.

\section{Bone marrow mononuclear cell isolation}

Mice were sacrificed by $\mathrm{CO}_{2}$ asphyxiation and femora, tibiae, pelves, humeri and vertebrae were crushed in phosphatebuffered saline (PBS). Red blood cells were lysed with $\mathrm{NH}_{4} \mathrm{Cl}$ in $\mathrm{PBS}$ and bone marrow mononuclear cells (BMMNC) were stained as described below.

\section{Flow cytometric analysis}

All flow cytometric analyses and sorting were performed on a BD FACS Aria II cell sorter. Antibodies used were CD4 (RM45, BD Biosciences), CD8a (53-6.7, eBioscience), B220 (RA36B2, Biolegend), CD11b (M1/70, BD Biosciences), Ly6G/C (RB6-8C5, Biolegend), Ter119 (TER-119, Biolegend), Sca1 (D7, Biolegend), MPL (AMM2, Immuno-Biological Laboratories), CD41 (MWReg30, BD Biosciences), CD150 (TC15-12F12.2, Biolegend), cKit (2B8, Biolegend), CD34 (RAM34, eBioscience), IL7R (A7R34, eBioscience), Flt3 (A2F10, eBioscience), CD16/32 (93, eBioscience) and CD105 (MJ7/18, Biolegend). Streptavidin (eBioscience) was used to resolve biotinylated
A

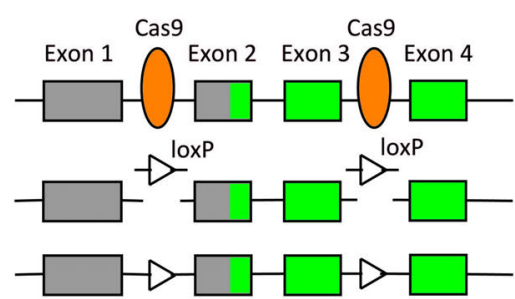

B

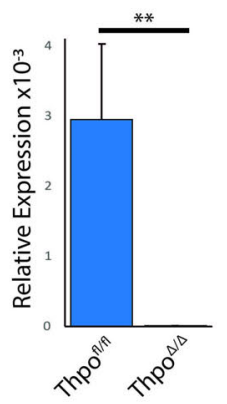

C
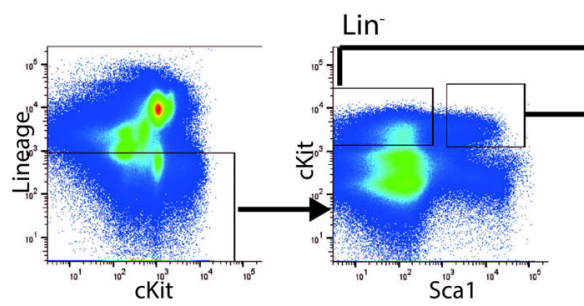

D
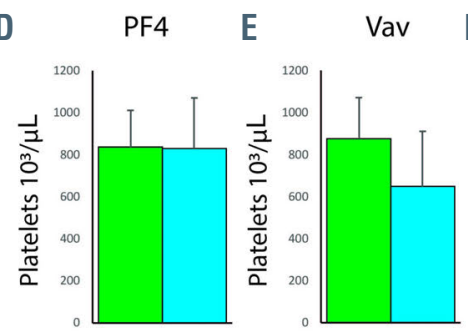

F

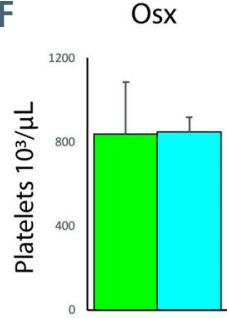

I
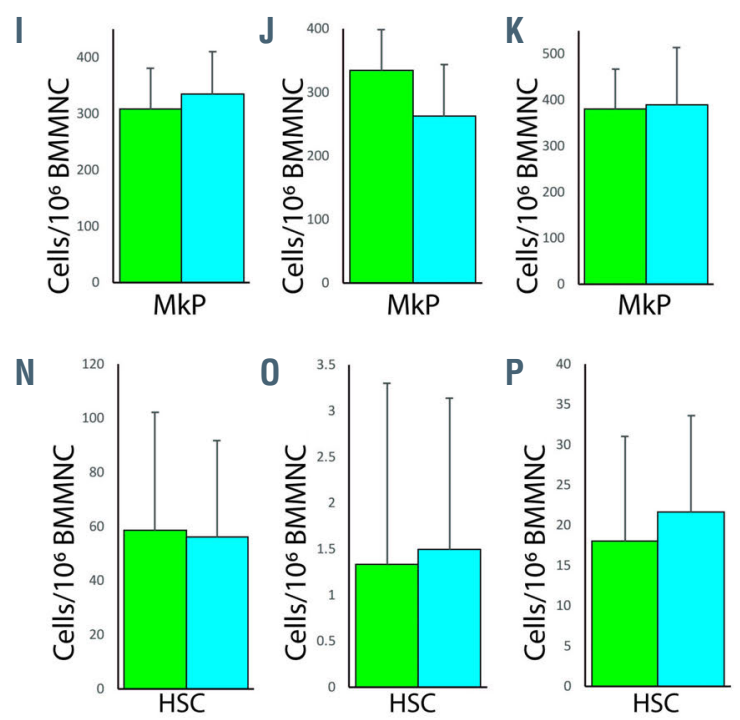

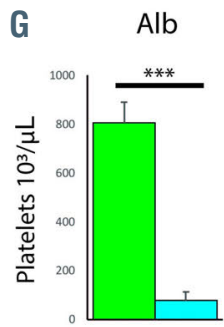

H

$\mathrm{KO}$
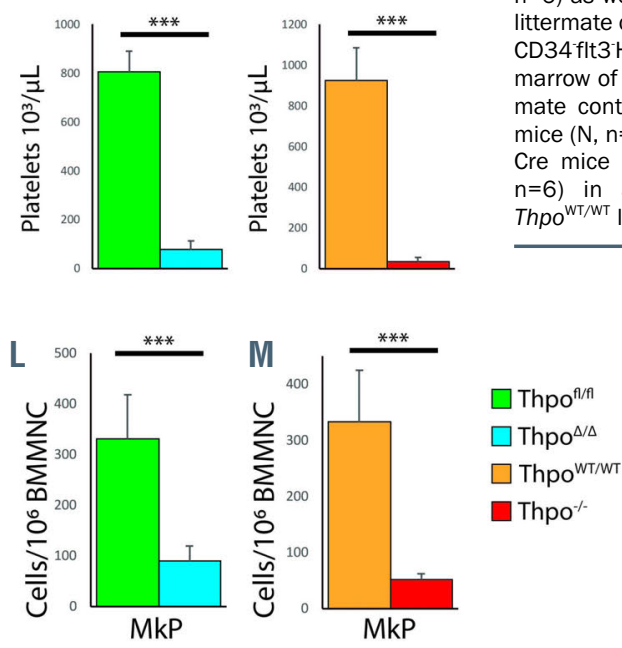

Figure 1. Thrombopoietin from liver maintains hematopoietic stem and progenitor cells in the bone marrow. (A) Schematic of Thpo flox generation. Two guide RNA were used to simultaneously cut two sites flanking exons 2 and 3 of the Thpo gene. Single-stranded deoxyribo-oligonucleotides were then used to insert loxP sequences by homology directed repair. (B) Quantitative polymerase chain reaction analysis of Thpo mRNA from livers of $T h p o^{\mathrm{fl} / \mathrm{fl}}$ and $T h p \mathrm{o}^{\Delta / \Delta \mathrm{Alb}-\mathrm{Cre}}$ mice relative to B2M expression $(n=3)$. (C) Flow cytometric gating system for analysis of hematopoietic stem cells (HSC) and megakaryocyte progenitors (MkP). Peripheral blood platelet counts were taken for Thpo ${ }^{\Delta / \Delta}$ mice and Thpof/fl littermate controls of PF4-Cre mice $\left(D, n=5^{\mathrm{t} / \mathrm{fl}} / 6^{\mathrm{s} / \Delta}\right)$, Vav-Cre mice $\left(E, n=5^{f / f l} / 6^{\Delta / \Delta}\right)$ Osx-Cre mice $(F$, $\left.\mathrm{n}=5^{\mathrm{ft} / \mathrm{fl}} / 6^{\Delta / \Delta}\right)$ and Alb-Cre mice $\left(\mathrm{G}, \mathrm{n}=5^{\mathrm{ft} / \mathrm{fl}}\right.$ $/ 6^{\Delta / \Delta}$ ) in addition to $T h p o /$ mice and Thpo ${ }^{\mathrm{WT} / \mathrm{NT}}$ littermate controls $\left(\mathrm{H}, \mathrm{n}=4^{\mathrm{WT} / \mathrm{WT}} / 6\right.$ '). Cell counts of MkP population in the bone marrow of $T h p o^{\Delta / \Delta}$ mice and $T h p o^{f / f l}$ littermate controls were analyzed in PF4Cre mice $(I, n=6)$, Vav-Cre mice $(J, n=6)$, Osx-Cre mice $(K, n=6)$ and Alb-Cre mice $(L$, $\mathrm{n}=6$ ) as well as Thpo-/mice and Thpo ${ }^{\mathrm{WT} / \mathrm{WT}}$ littermate controls $(M, n=6)$. Cell counts of CD34flt3 HSC populations in the bone marrow of $T h p o^{\Delta / \Delta}$ mice and $T h p o^{f / f / l}$ littermate controls were analyzed in PF4-Cre mice $(N, n=6)$, Vav-Cre mice $(0, n=6)$, OsxCre mice $(P, n=6)$ and Alb-Cre mice $(Q$, $\mathrm{n}=6$ ) in addition to Thpo/mice and Thpo ${ }^{\text {WT }}{ }^{\mathrm{WT}}$ littermate controls $(\mathrm{R}, \mathrm{n}=6)$.
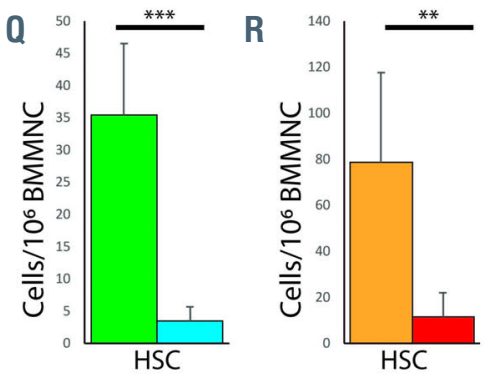
antibodies and propidium iodide was added prior to analysis to identify live cells.

\section{mRNA expression}

Cells were sorted into Trizol (Thermo Fisher Scientific) and RNA was isolated according to the manufacturer's protocol. Complementary DNA was reverse transcribed by a SuperScript VILO cDNA Synthesis Kit (Thermo Fisher Scientific) and mRNA expression analyzed using TaqMan Fast Advanced Master Mix (Thermo Fisher Scientific) in a QuantStudio 3 Real-Time PCR system (Thermo Fisher Scientific). Expression levels were calculated relative to the expression of Gapdh.

\section{In vitro culture}

BMMNC were isolated as above and cKit enrichment was performed using magnetic activated cell sorting (MACS)-conjugated cKit antibodies (Miltenyi Biotec) and an AutoMACS cell sorter according to the manufacturer's protocol. Enriched cells were stained with antibodies and sorted to StemSpanII (StemCell Technologies) containing stem cell factor (SCF, $20 \mathrm{ng} / \mathrm{mL}$, Peprotech) and THPO (20 ng/mL, Peprotech), or SCF, THPO, interleukin-3 (IL3, 20 ng/mL, Peprotech), interleukin-6 (IL6, 20 $\mathrm{ng} / \mathrm{mL}$, Peprotech) and erythropoietin (EPO, $10 \mathrm{ng} / \mathrm{mL}, \mathrm{R} \& \mathrm{D}$ Systems). On day 10 of culture colonies were stained with CD41, CD16/32, CD71 (C2, BD Biosciences), Ter119, CD11b,
A

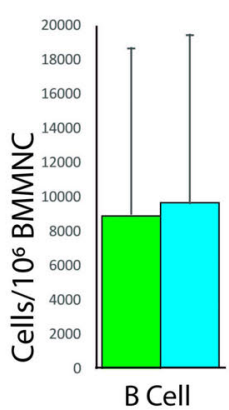

E

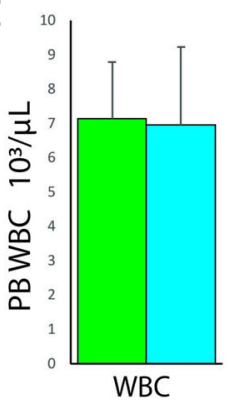

B

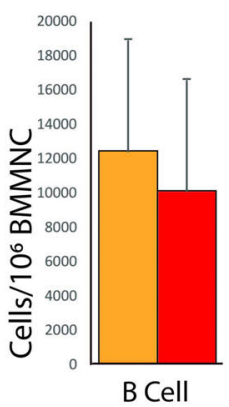

$\mathbf{F}$

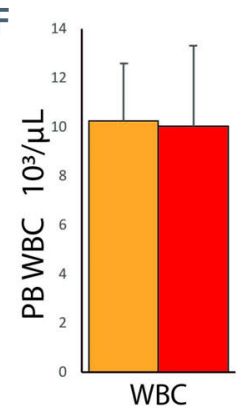

C

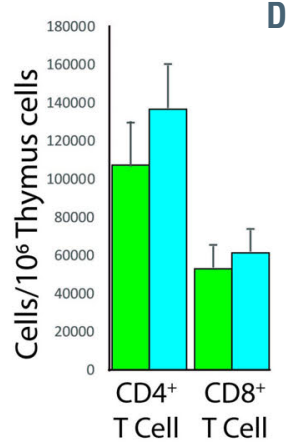

G

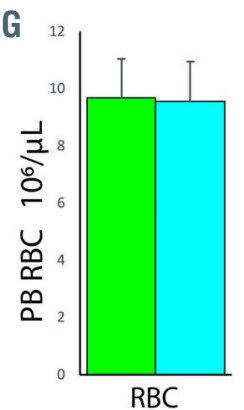

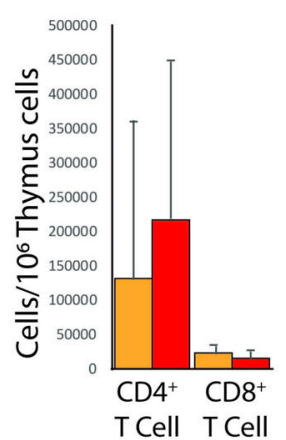

H

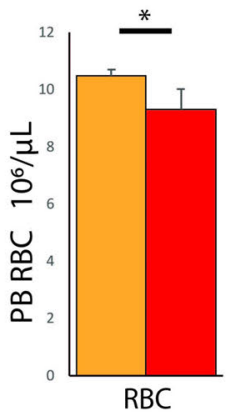

I

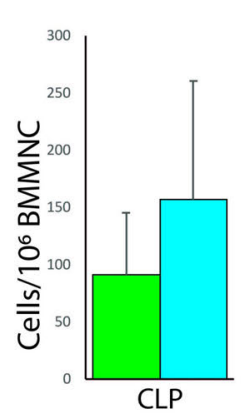

J

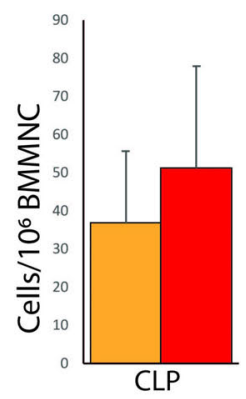

K

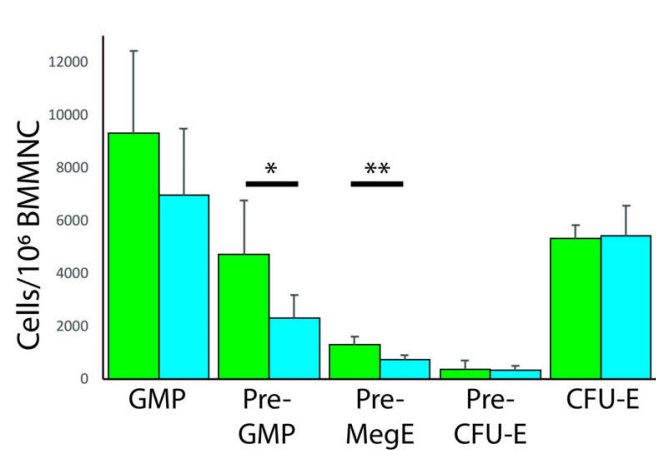

L

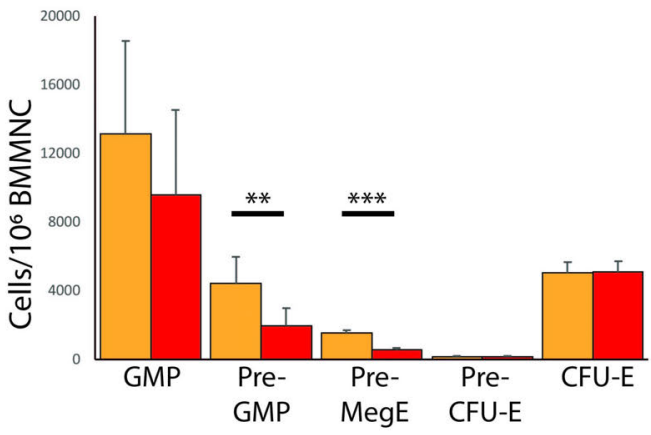

$\square T_{p p o}^{\text {f/f }}$

$\square \operatorname{Thpo}^{\Delta / \Delta}$

$\square$ Thpo ${ }^{\text {WTWT }}$

DThpo ${ }^{--}$
Figure 2. Thrombopoietin maintains hematopoietic stem and progenitor cells with megakaryopoietic potential. (A, B) Mature B-cell counts were analyzed in the bone marrow of $T h p o^{\Delta / \Delta A l b-C r e}$ and $T h p o^{f / f l}$ control mice (A), as well as Thpo ${ }^{--}$and Thpo ${ }^{\mathrm{WT} / \mathrm{WT}}$ mice (B). (C, D) Thymus $\mathrm{CD} 4^{+}$and $\mathrm{CD}^{+} \mathrm{T}$ cell numbers were analyzed in the two models. $(E-H)$ Peripheral blood white blood cells $(E, F)$ and red blood cells $(G, H)$ were quantified. (I-L) Lymphoid progenitors (I, J) and myeloid progenitors $(\mathrm{K}, \mathrm{L})$ were quantified In the bone marrow of the two murine models. $(n=6)$ PB: peripheral blood; WBC: white blood cells; RBC: red blood cells; BMMNC: bone marrow mononuclear cells; CLP: common lymphoid progenitors; GMP: granulocyte/ macrophage progenitors; MegE: megakaryocute/erythroid progenitors; CFUE: colony-forming unit erythroid. 
Ly6C/G and propidium iodide was added to identify live cells. Lineages were gated as shown in Online Supplementary Figure S1 and colonies were defined by the presence of $>20$ cells of any one of each lineage.

\section{Cell cycle analysis}

cKit-enriched BMMNC were isolated as above and stained with PerCP-Cy5.5 lineage cocktail, FITC-conjugated CD34, PEconjugated CD41, PE-Cy7-conjugated Sca1, APC-conjugated CD150 and APC-Cy7-conjugated cKit. Cells were then fixed and permeabilized by IntraPrep Reagent (Beckman Coulter) in accordance with the manufacturer's protocol. AlexaFluor700-conjugated Ki67 (SolA15, Invitrogen) antibodies and Hoechst 33342 were added after permeabilization and cells were analyzed by flow cytometry.

\section{Rescue experiments}

For the rescue experiments, $1 \mathrm{mg}$ CXCL4/PF4 (Peprotech) or PBS was injected intraperitoneally daily for 7 days and bone marrow analyzed on day 7. Romiplostim (Kyowa Kirin) or PBS as a control was administered intravenously at a dose of 100 $\mu \mathrm{g} / \mathrm{kg}$ per day for 5 days.

\section{Statistical analysis}

All values given are mean \pm standard deviation. Statistical analyses were performed by two-tailed unpaired $t$-tests and the
A

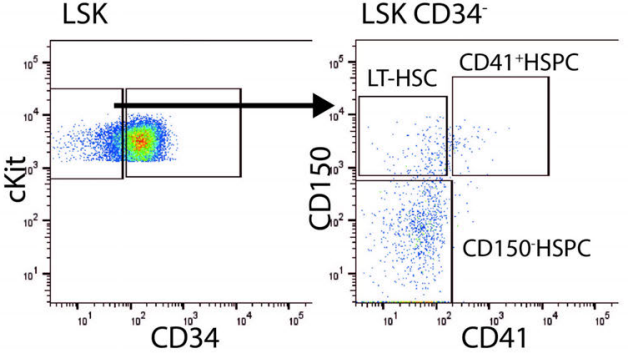

B

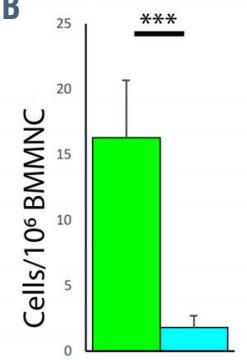

LT-HSC

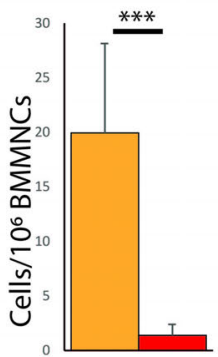

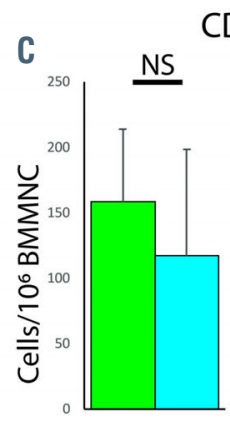

CD150-HSPC
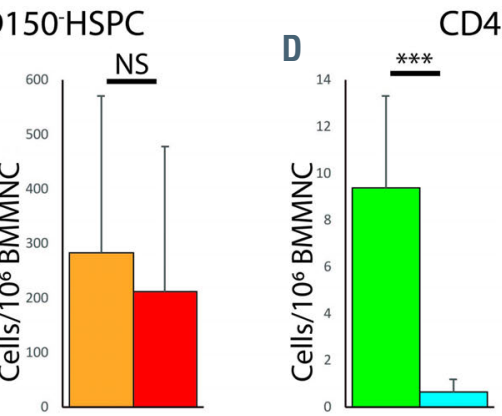

CD41+HSPC
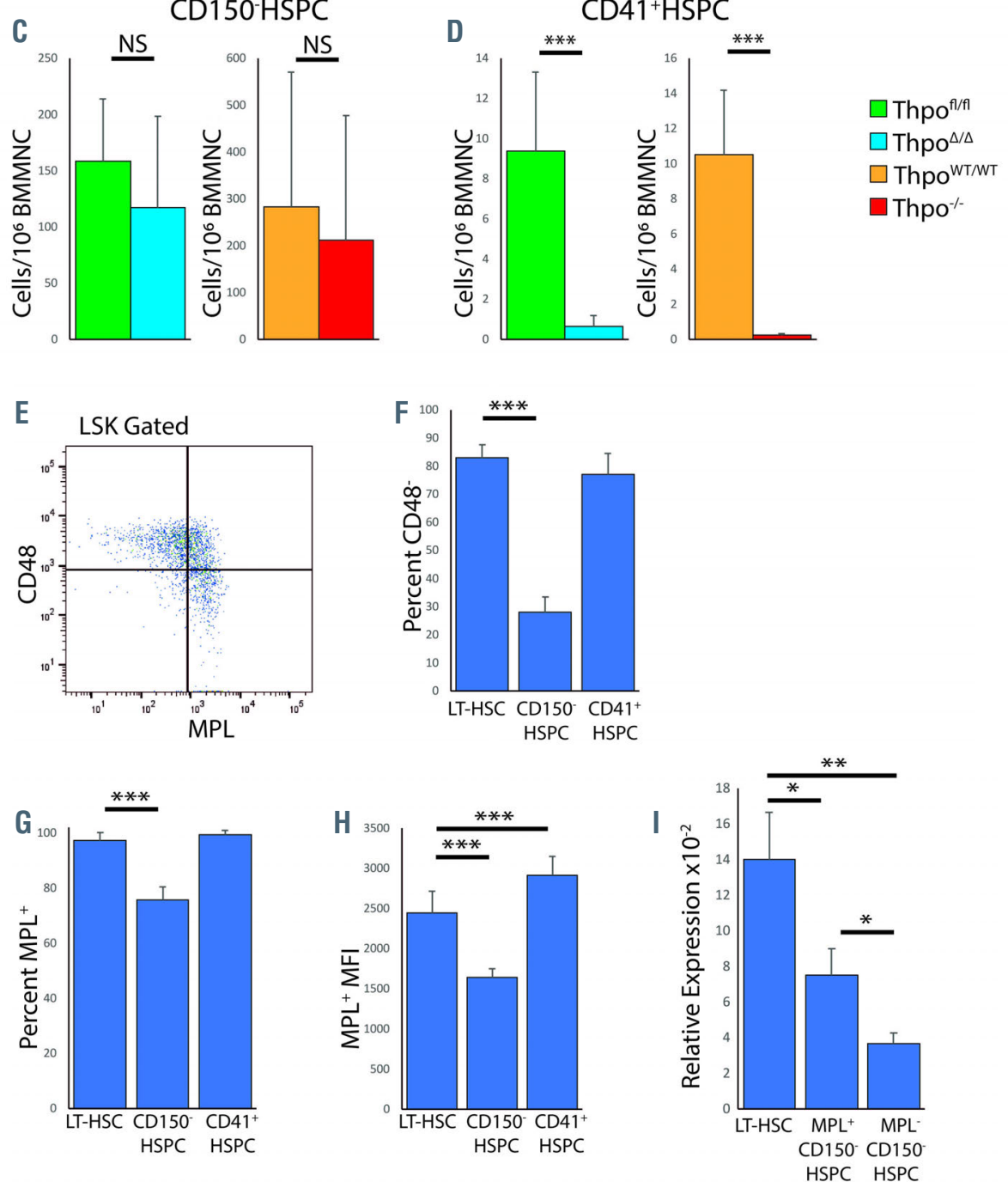

Figure 3. Hematopoietic stem and progenitor cells show variable responses to loss of thrombopoietin/MPL signaling. (A) Flow cytometric gating system for hematopoietic stem and progenitor cell (HSPC) populations. (B-D) Cell counts of populations in bone marrow

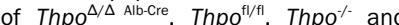
Thpo ${ }^{\mathrm{WT} / \mathrm{WT}}$ mice were analyzed for longterm hematopoietic stem cells (LT-HSC) (B), CD150- HSPC (C) and CD41 ${ }^{+}$HSPC (D) $(n=6)$. (E) A representative plot of CD48 and MPL expression in LSK cells, with CD48 and MPL gating lines indicated. (F) Percentage of wild-type (WT) cells from each HSPC population that are $\mathrm{CD}^{-} 8^{-}(\mathrm{n}=8)$. (G) Percentage of CD48 cells from each population expressing MPL $(n=8)$. (H) MPL mean fluorescence intensity (MFI) of CD48$\mathrm{MPL}^{+}$cells from each population $(n=8)$. (I) MPL mRNA expression levels relative to GAPDH $(n=3)$. BMMNC: bone marrow mononuclear cells. 
levels of statistical significance are represented by asterisks. ${ }^{*} P<0.05,{ }^{* *} P<0.01,{ }^{* * *} P<0.001$. NS represents $P>0.05$.

\section{Results} Hematopoietic stem and progenitor cells in bone mar-
row require thrombopoietin from liver

To investigate the role of THPO in HSPC we first sought the source of THPO in the bone marrow. We generated a Thpo ${ }^{\text {flox }}$ mouse using CRISPR/Cas9 to simultaneously insert two loxP sites flanking exons 2 and 3 of the Thpo gene (Figure 1A). Loss of Thpo mRNA expression in the liver of Thpo $0^{\Delta \Delta \mathrm{Alb}-\text {-Cre }}$ mice was confirmed by quantitative polymerase chain reaction analysis (Figure 1B). In agreement with Decker et al. ${ }^{11}$ we found that liver-specific knockout (KO) of THPO caused similar loss of platelets to that of $\mathrm{Thpo}^{-1}$, while conditional $\mathrm{KO}$ models for megakaryo-cytes, HSPCs and bone marrow stromal cells (PF4, Vav and Osx respectively) did not show any significant difference (Figure 1D-H). ${ }^{11}$ Similarly, the numbers of bone marrow MkP were significantly reduced in Thpo ${ }^{\Delta / \Delta}$ Alb-Cre mice and Thpo ${ }^{-/}$mice, but not in other conditional KO models (Figure 1I-M). We also observed a similar pattern in the CD34-FIt3-LSK HSC population, with a reduction in cell numbers within the bone marrow of Thpo $0^{\Delta \Delta \mathrm{Alb}-}$ ${ }^{C r e}$ mice similar to that of $\mathrm{Thpo}^{-/-}$mice, but no reduction in bone marrow-specific conditional KO models (Figure 1N-
R). This would suggest that steady-state HSPC in the bone marrow mainly depend on THPO from the liver.

\section{Thromobopoietin maintains megakaryopoietic progeni- tor numbers}

We set out to find which other hematopoietic cells are influenced by THPO. Analysis of mature blood lineages revealed that $\mathrm{B}$ cells in the bone marrow (Figure $2 \mathrm{~A}$ and B), T cells in thymus (Figure 2C and D) and white blood cells in peripheral blood (Figure 2E and F) were unaffected by loss of THPO in both Thpo $0^{\text {f/flallb-Cre }}$ and Thpo ${ }^{-/}$models. No effect on peripheral blood red blood cells was seen in Thpo $0^{\mathrm{f} / \mathrm{A} / \mathrm{Al} \text {-Cre }}$ mice and although statistical analysis showed a significant reduction in Thpo ${ }^{-1}$ mice, this appears to be very low and may simply be an artefact of variance within the mouse strain (Figure $2 \mathrm{G}$ and $\mathrm{H}$ ). To confirm that the loss of THPO did not affect mature lineages other than megakaryocytes, progenitor populations in bone marrow were analyzed. Common lymphoid progenitors in the bone marrow were unaffected by loss of THPO in both models (Figure 2I and J). Analysis of myeloid progenitors showed that only the previously defined Pre-GMP and Pre-MegE populations were affected by the loss of THPO (Figure $2 \mathrm{~K}$ and L). Interestingly, these populations were previously shown to give rise to megakaryocytes in vitro while the other myeloid progenitors did not show megakaryopoietic potential, ${ }^{12}$ suggesting that only the MkP, Pre-GMP and
A

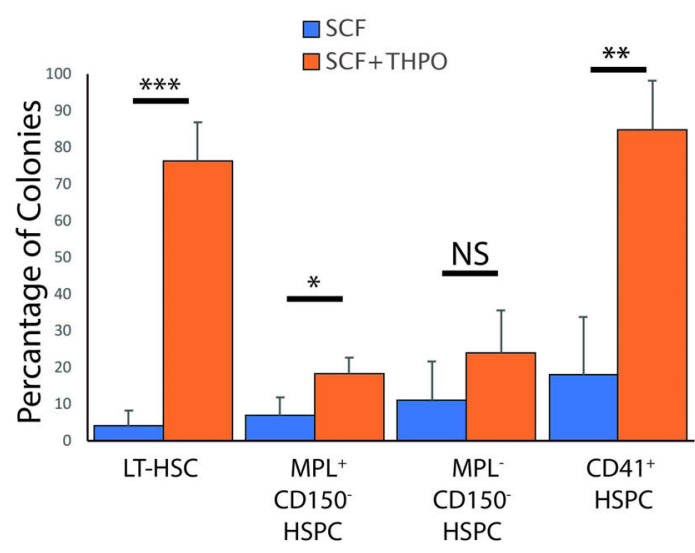

B

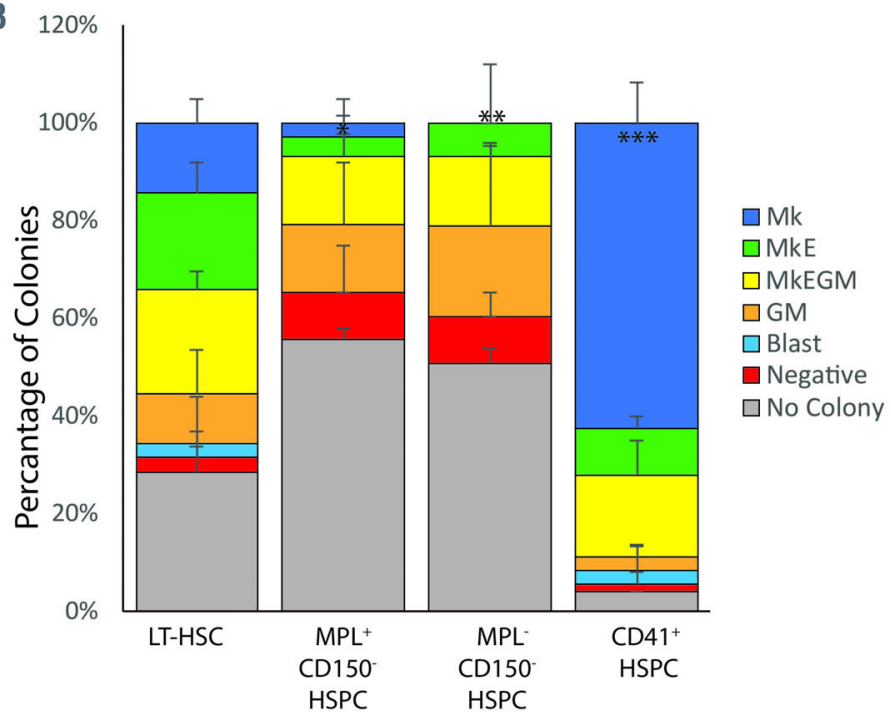

C

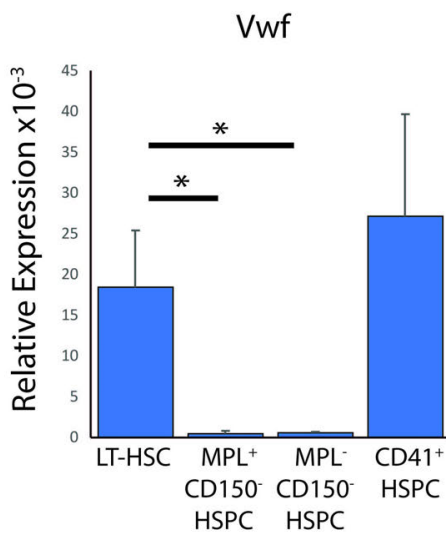

D

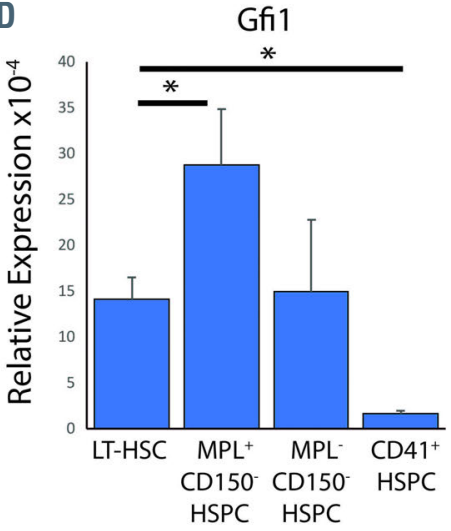

Figure 4. CD150 hematopoietic stem and progenitor cells have reduced thrombopoietin dependence and low megakaryopoietic potential. (A) Single cells of each hematopoietic stem and progenitor cell (HSPC) population were cultured in vitro with stem cell factor (SCF) or SCF and thrombopoietin (THPO) and colonies were counted on day 10. Bars show the percentage of sorted cells that gave rise to colonies ( $n=72$ single cells across 3 experiments). (B) Single cells of each HSPC population were cultured in vitro and colonies analyzed by fluorescence activated cell sorting on day 10. Bars show the percentage of colonies that contain specific lineages ( $n=72$ single cells across 3 experiments). (C, D) mRNA expression of Vwf (C) and Gfi1 (D) relative to GAPDH in HSPC $(n=3)$. LT-HSC: long-term hematopoietic stem cell, Mk: megakaryocyte; MkE: megakaryocyte-erythroid; MkEGM megakaryocyte-erythroid-granulocyte-macrophage; GM: granulocyte-macrophage. 
Pre-MegE cells that give rise to megakaryocytes are affected by the loss of THPO. Analysis of MPL expression among these progenitor populations suggests that, while MPL was detectable in all populations, most cell populations showed baseline levels. Only those populations with megakaryocytic potential showed levels of expression significantly above the baseline (Online Supplementary Figure S2).

\section{Thrombopoietin is required to maintain $\mathrm{CD}_{150^{+}}$ hematopoietic stem cell numbers}

To further analyze the hematopoietic compartment the CD34-LSK HSC fraction was subdivided into three HSC populations based on CD41 and CD150 expression (Figure 3A). Previous studies have defined the CD34 CD $41^{-} \mathrm{CD} 150^{+}$LT-HSC population as being enriched for long-term repopulating HSC, while the CD150- HSPC population contains short-term repopulating HSC and lymphoid-biased HSC. ${ }^{13,14}$ The CD $41^{+}$HSPC population has been proposed to be enriched for common myeloid progenitors with the potential to repopulate the bone marrow of lethally irradiated mice. ${ }^{15}$ This method of defining populations was found to be optimal for distinguishing those LSK populations that express high levels of MPL from those with reduced MPL expression (Online Supplementary Figure S3) as well as for separating populations based on differentiation potential. Analysis revealed that the two $\mathrm{CD} 150^{+}$LSK populations, LT-HSC and CD $41^{+}$HSPC, showed significant loss of cell numbers with LT-HSC numbers reduced from $16.3 \pm 4.35$ cells per $10^{6}$ bone marrow mononuclear cells (BMMNC) to $1.8 \pm 0.89$ in $T h \mathrm{po}^{\Delta / \Delta \mathrm{Alb}-\mathrm{Cre}}$ mice and from $19.9 \pm 8.21$ to $1.4 \pm 0.94$ in $\mathrm{Thpo}^{-/-}$mice (Figure 3B). CD $41^{+} \mathrm{HSPC}$ also decreased drastically from $9.4 \pm 3.92$ to $0.7 \pm 0.52$ in $\mathrm{Thpo}^{\Delta / \Delta}$ Alb-Cre mice and from $10.5 \pm 3.68$ to $0.2 \pm 0.08$ in $\mathrm{Thpo}^{-/}$mice, a reduction of over $90 \%$ (Figure 3D). The CD150 HSPC population, however, showed no significant difference in either Thpo ${ }^{\mathrm{fl} / \mathrm{fl} A \mathrm{llb}-\mathrm{Cre}}$ or Thpo-/ mice, suggesting that this population may have reduced dependence on THPO signaling (Figure 3C).

\section{CD150 hematopoietic stem and progenitor cells have reduced MPL expression}

As CD48 is expressed by lineage-committed cells we analyzed CD48 expression in the HSPC populations. While a high proportion of LT-HSC and CD $41^{+} \mathrm{HSPC}$ did not express CD48, the majority of CD150 HSPC were $\mathrm{CD}_{4}{ }^{+}$(Figure $3 \mathrm{E}$ and $\mathrm{F}$ ). To investigate why the two $\mathrm{CD} 150^{+}$populations were highly affected by loss of THPO signaling while CD150- HSPC were not, we looked at the surface expression of MPL of the three populations. The CD48 CD150- HSPC expressed MPL, but a significantly smaller proportion of these cells expressed MPL compared to the other two HSC populations (Figure 3G). Among the $\mathrm{MPL}^{+} \mathrm{CD} 48^{-} \mathrm{CD} 150^{-} \mathrm{HSPC}$ the MPL mean fluorescence intensity was lower than in $\mathrm{MPL}^{+} \mathrm{CD} 48^{-} \mathrm{LT}-\mathrm{HSC}$, suggesting that MPL is being downregulated as LT-HSC differentiate into $\mathrm{CD}^{150^{-}} \mathrm{HSPC}$ (Figure $3 \mathrm{H}$ ). To confirm that MPL expression is reduced

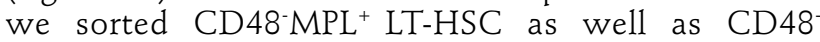
$\mathrm{MPL}^{+} \mathrm{CD} 150^{-} \mathrm{HSPC}$ and $\mathrm{CD}^{-} 8^{-} \mathrm{MPL}^{-} \mathrm{CD} 150^{-} \mathrm{HSPC}$ and analyzed MPL mRNA expression (Figure 3I). We observed that even in the $\mathrm{MPL}^{+} \mathrm{CD} 150^{-} \mathrm{HSPC}, \mathrm{MPL}$ mRNA expression was significantly reduced compared to that in LT-HSC and was further reduced in MPL CD150- HSPC. This suggests that MPL is downregulated as LT-HSCs differentiate into CD150 HSPC and that CD150-HSPC may therefore be less dependent on THPO/MPL signaling. Analysis of MPL expression in the $\mathrm{KO}$ models showed that smaller proportions of LT-HSC and CD150- HSPC were expressing MPL in both Thpo-

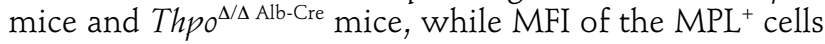

A
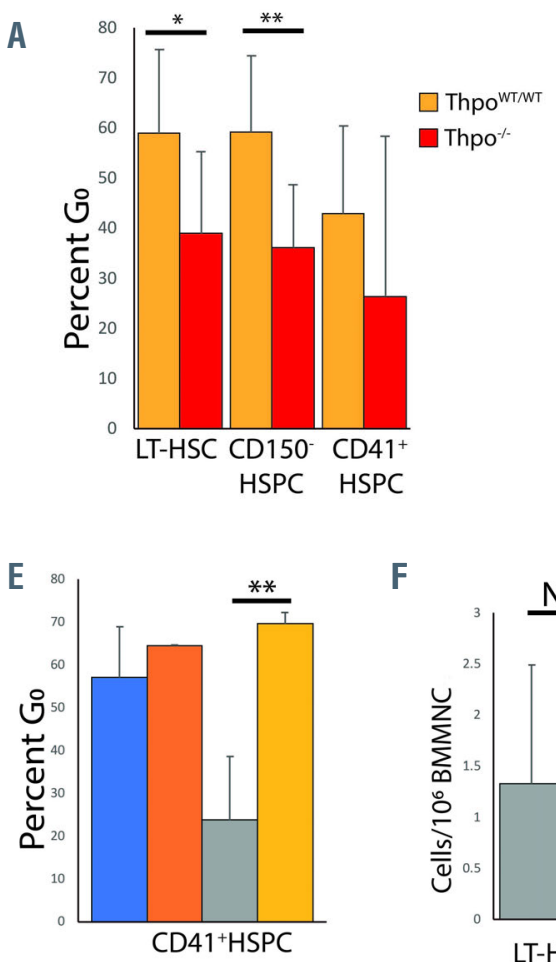

B

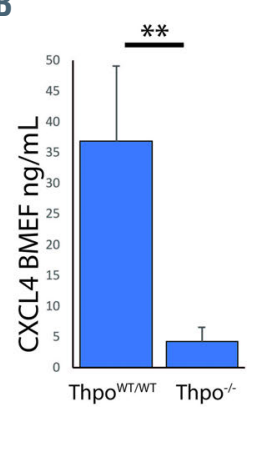

$\mathbf{F}$

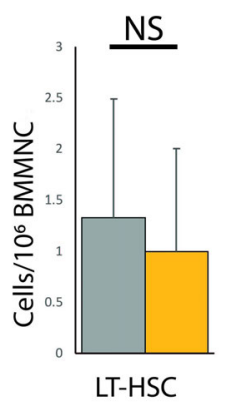

C

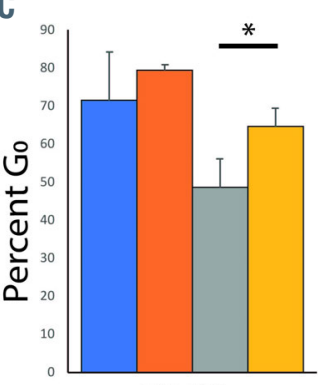

LT-HSC

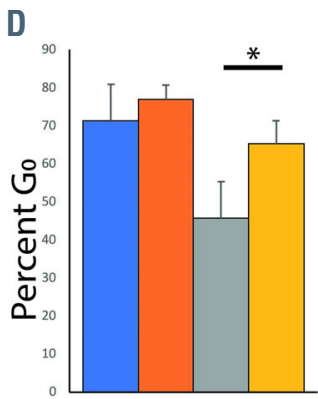

CD150-HSPC

Figure 5. Maintenance of cell number is independent of quiescence. (A) Quiescence was measured as the percentage of $\mathrm{Ki} 67$ negative $\left(\mathrm{G}_{0}\right)$ cells within hematopoietic stem and progenitor cell (HSPC) populations of Thpo $\%$ and Thpo ${ }^{\text {WT } / W T}$ control mice. (B) The concentration of CXCL4/PF4 in bone marrow of knockout mice as measured by enzyme-linked immunosorbent assay $(n=4)$. (C-E) Percentage of cells in $\mathrm{G}_{\text {。 }}$ at day 7 of intraperitoneal treatment with $\mathrm{CXCL4/PF4}$ or phosphatebuffered saline (PBS) in $\mathrm{Thpo}^{\%}$ mice and Thpo $^{\text {WT/WT }}$ littermate controls $(n=3)$. ( $\left.F, G\right)$ Cell counts of HSPC populations at day 7 of intraperitoneal treatment with CXCL4/PF4 or PBS in Thpo1. mice and Thpo ${ }^{\text {WT/WT }}$ littermate controls $(n=3)$. BMEF: bone marrow extracellular fluid; BMMNC: bone marrow mononuclear cells. 
was not affected (Online Supplementary Figure S4). This suggests specific loss of $\mathrm{MPL}^{+}$cells in the absence of THPO. Despite this, MPL expression in CD $41^{+}$HSPC was unaffected by loss of THPO, suggesting that this population of HSPC is more homogeneous than the other two HSPC populations and that MPL expression is intrinsic to $\mathrm{CD} 41^{+} \mathrm{HSPC}$.

\section{CD150 hematopoietic stem and progenitor cells have reduced dependence on thrombopoietin and low megakaryopoietic potential}

To examine whether the CD150- HSPC are dependent on THPO signaling for proliferation, single $\mathrm{MPL}^{+} \mathrm{CD} 48$ CD150 HSPC and MPL-CD48 CD150 HSPC were cultured with THPO and SCF for 7 days. Proliferation of CD48 $\mathrm{MPL}^{+}$LT-HSC and CD41 ${ }^{+} \mathrm{HSPC}$ was also analyzed (Figure 4A). A high proportion of the LT-HSC and CD41 ${ }^{+}$ HSPC gave rise to colonies at day 10 in the presence of THPO, but in the absence of THPO few colonies were observed. This indicates that the two $\mathrm{CD} 150^{+}$populations are dependent on THPO for proliferation in vitro. Both $\mathrm{MPL}^{+} \mathrm{CD}_{150}^{-} \mathrm{HSPC}$ and $\mathrm{MPL}^{-} \mathrm{CD} 150^{-} \mathrm{HSPC}$ produced few colonies in the presence of THPO and while the $\mathrm{MPL}^{+} \mathrm{CD} 150^{-} \mathrm{HSPC}$ produced slightly fewer colonies in the absence of THPO, the colony number among MPL CD150- HSPC was not significantly different. This suggests that the CD150 HSPC population has reduced dependence on THPO for proliferation in vitro, with the
MPL'CD150- HSPC population showing limited dependence on THPO/MPL signaling. Downregulation of MPL expression has previously been linked to lymphoid lineage commitment, ${ }^{16}$ suggesting that the CD150 HSPC would have reduced myeloid differentiation potential compared to the other LSK populations. To test this we utilized an adapted method of a previously described in vitro culture assay. ${ }^{17}$ The $\mathrm{CD} 48$ fractions of each population were cultured with SCF, THPO, EPO, IL3 and IL6 to assess their differentiation potentials (Figure 4B). At day 10 fewer than $50 \%$ of single cells from the two CD 150 HSPC populations produced colonies, suggesting that the myeloid differentiation cytokines in the media were insufficient to support the proliferation of many of the cells in these populations. Both $\mathrm{MPL}^{+} \mathrm{CD} 48^{-} \mathrm{CD} 150^{-} \mathrm{HSPC}$ and MPL ${ }^{-}$CD48 ${ }^{-} \mathrm{CD} 150^{-} \mathrm{HSPC}$ populations produced significantly fewer Mk colonies than LT-HSC and CD41 ${ }^{+}$ HSPC. Previous studies have shown that MPL is downregulated as HSC become lymphoid-biased. Taken together this suggests that megakaryopoietic potential is lost as the CD150 HSPC become lymphoid-primed. Over $50 \%$ of $\mathrm{CD}^{4} \mathrm{MPL}^{+} \mathrm{LT}-\mathrm{HSC}$ gave rise to colonies containing megakaryocytes, while approximately $90 \%$ of CD 48 $\mathrm{MPL}{ }^{+} \mathrm{CD} 41^{+} \mathrm{HSPC}$ give rise to colonies with megakaryocytes. Subsequent mRNA expression analysis revealed that while LT-HSC and CD $41^{+}$HSPC both highly expressed the megakaryocyte marker, von Willebrand factor (Vwf), the two CD150- HSPC populations showed

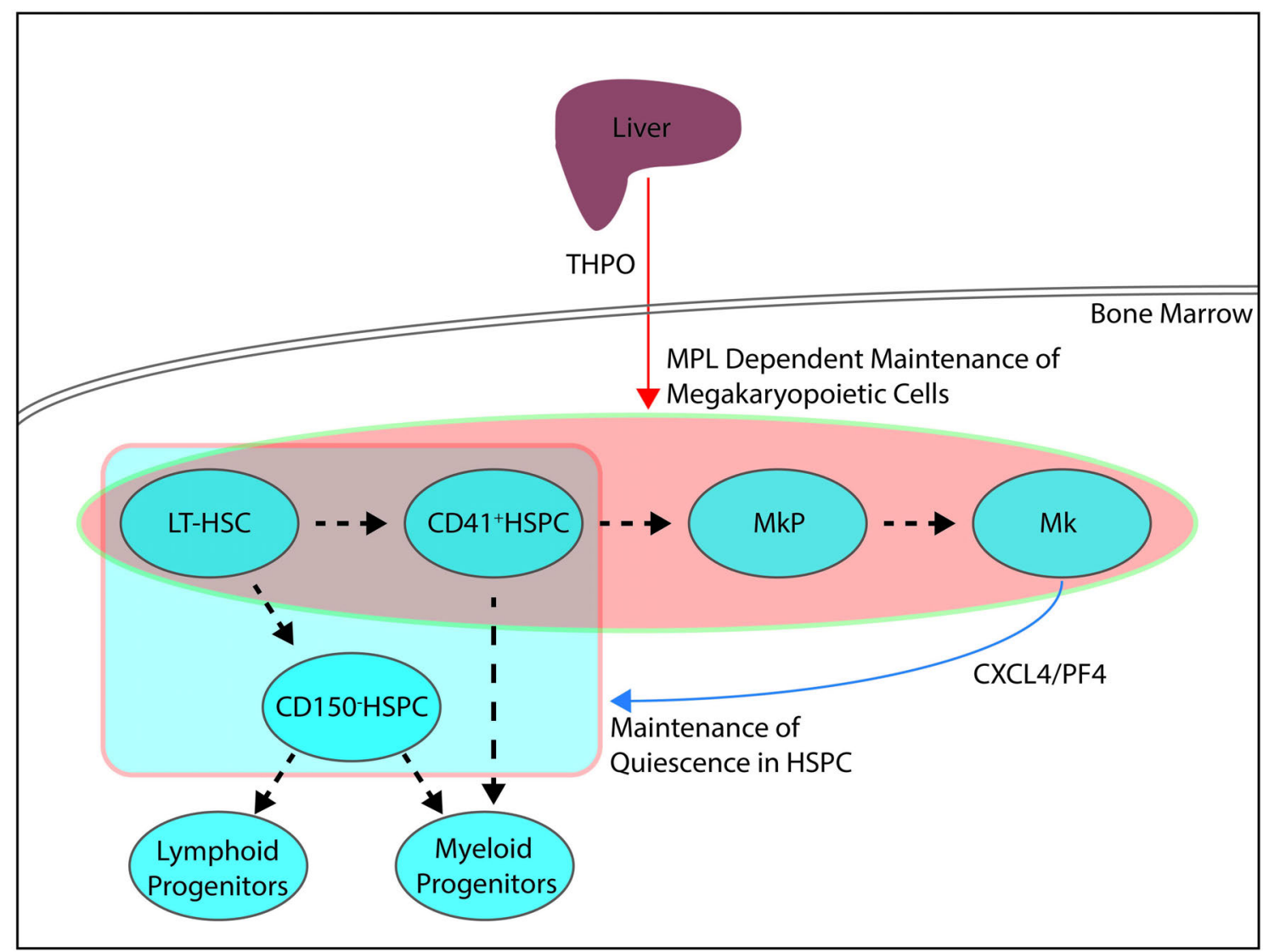

Figure 6. Proposed model of maintenance of megakaryopoietic hematopoietic stem and progenitor cell number and quiescence. Thrombopoietin (THPO) from liver maintains megakaryopoietic cell numbers in the bone marrow. This preserves homeostatic levels of megakaryocyte-derived CXCL4, which induces quiescence in hematopoietic stem and progenitor cell (HSPC) populations. CD150 HSPC rely on CXCL4 for quiescence but are not dependent on THPO for maintenance of cell numbers. LT-HSC: long-term hematopoietic stem cell; MkP: megakaryocyte progenitor; MK: megakaryocyte. 
significantly lower expression, indicative of their reduction in megakaryopoietic potential (Figure 4C). Analysis of the expression of the neutrophil/lymphoid marker (Gfi1) revealed that the expression of Gfil was higher in the CD150 HSPC population than in LT-HSC, and its expression was reduced in $\mathrm{CD}_{4} 1^{+} \mathrm{HSPC}$ (Figure 4D). These data suggest that the CD150- HSPC may be lymphoid-primed at the expense of megakaryopoietic potential, while the CD41 $1^{+} \mathrm{HSPC}$ may become megakaryocyteprimed at the expense of lymphoid potential.

\section{Loss of quiescence is not responsible for loss of cell number}

Previous studies have shown that THPO plays a role in maintaining HSC quiescence. ${ }^{4}$ In order to investigate this, we first looked at the quiescence levels of the HSC populations in $\mathrm{Thpo}^{-/}$mice (Figure 5A). The frequency of quiescence was reduced in all three HSPC populations. Interestingly, quiescence was reduced in CD150- HSPC despite them having limited dependence on THPO/MPL signaling. Previous studies have suggested that megakaryocytes in the bone marrow are responsible for maintaining HSCs in quiescence through the megakaryocyte-specific cytokine CXCL4/PF4. ${ }^{18}$ We looked at CXCL4/PF4 levels in the bone marrow of $\mathrm{Thpo}^{-/}$mice and found that CXCL4/PF4 concentration is decreased on loss of THPO (Figure 5B). This suggests that the loss of megakaryocytes in $\mathrm{KO}$ mice reduces bone marrow CXCL4/PF4 concentration. To assess whether administration of recombinant CXCL4/PF4 could rescue quiescence in $\mathrm{Thpo}^{-/}$mice, CXCL4/PF4 was injected daily for 7 days. Treated KO mice showed a significant increase in the proportion of cells in $\mathrm{G}_{0}$ in all three HSPC populations (Figure 5C-E). The quiescence levels in treated $\mathrm{KO}$ mice were not significantly different from those of wild-type untreated mice, suggesting that CXCL4/PF4 treatment rescues quiescence back to wild-type levels in all three HSPC populations. Taken together these data would suggest that the HSPC require CXCL4/PF4 from megakaryocytes for maintenance of quiescence. Although quiescence is rescued by CXCL4/PF4 treatment the number of LT-HSCs and CD150- HSPC remained unchanged (Figure 5F and G). This would suggest that the loss of cell numbers is independent of loss of quiescence. To confirm this we treated $\mathrm{Thpo}^{-/}$mice with the MPL receptor agonist romiplostim for 5 days via tail vein injection. On day 5 platelet counts in $\mathrm{Thpo}^{-/}$mice had recovered to the level in wild-type untreated mice, suggesting that thrombopoietic potential - and by extension megakaryopoietic potential - is not lost in the absence of THPO signaling and can return to wild-type levels upon rescue of MPL signaling (Online Supplementary Figure S5A).

\section{Discussion}

We have shown that loss of THPO signaling in bone marrow leads to a reduction of cell numbers throughout the hematopoietic compartment, primarily in cells that express high levels of MPL and show potential for megakaryopoietic differentiation. We show that the CD150- HSPC population has low MPL expression and reduced megakaryopoietic potential, findings that are consistent with previous reports that these cells are lymphoid-biased..$^{14,16}$ Previous reports have defined the $\mathrm{CD} 150^{-} \mathrm{HSPC}$ and $\mathrm{CD} 41^{+} \mathrm{HSPC}$ populations as HSC, although strictly speaking these populations contain predominantly hematopoietic progenitor cells, rather than true HSCs. In spite of this, it is important to note that these populations are both capable of repopulating the bone marrow of lethally irradiated mice, though not long-term, indicating a capacity for self-renewal. Both CD41 $1^{+}$HSPC and CD150- HSPC populations also show high levels of quiescence similar to LT-HSC and express cell surface markers very similar to those of LT-HSC, suggesting a very close relationship between the three populations. Indeed, previous studies have suggested that CD $41^{+} \mathrm{HSPC}$ and CD150 HSPCs represent the earliest branch point in the hematopoietic hierarchy, with $\mathrm{CD} 41^{+} \mathrm{HSPC}$ being the earliest myeloid branch and CD150 ${ }^{\circ} \mathrm{HSPC}$ being the earliest lymphoid branch. ${ }^{14}$ The data in this study support this view.

Despite their low levels of MPL expression and limited dependence on THPO for cell proliferation both in vivo and in vitro, CD150 HSPC show reduced quiescence in the absence of THPO in the bone marrow. Previous studies showed that loss of megakaryocytes in bone marrow results in reduced HSC quiescence, ${ }^{19,20}$ while a subsequent study showed that this effect is due to loss of megakaryocyte-derived CXCL4/PF4. ${ }^{18}$ We show that in the case of THPO KO mouse models, the loss of quiescence in HSPC results from the loss of CXCL4/PF4 signaling due to reduced megakaryocyte number in the bone marrow and that this can be rescued by administration of exogenous CXCL4/PF4. Interestingly, CXCL4/PF4 administration in wild-type mice does not produce an increase in quiescence, suggesting that quiescence is not dependent on CXCL4/PF4 signaling alone. Previous studies have shown that CXCL12 from CXCL12-abundant reticular (CAR) cells in the bone marrow also plays a role in HSPC quiescence. ${ }^{21}$ Other studies have provided evidence of dimerization of CXC ligands, including CXCL12. ${ }^{22}$ One theory is that CXCL4/PF4 and CXCL12 form heterodimers that induce quiescence in $\mathrm{HSC}$ and that without an increase in CXCL12, increased CXCL4/PF4 cannot induce further quiescence. Further research may clarify whether CXCL4/PF4-dependent quiescence is co-dependent on other signaling pathways.

Our in vitro and in vivo data suggest that MPL expression in HSC correlates closely with megakaryocytic differentiation potential, indicating that THPO in the bone marrow is responsible for maintaining megakaryocytic differentiation potential in HSPC. A recent study from our own group has shown that increased THPO/MPL signaling leads to increased proliferation and megakaryocytic differentiation as well as mitochondrial activation in HSCs, suggesting that THPO drives cell division, rather than suppressing it. ${ }^{23}$ There are reports that THPO induces self-renewal division in $\mathrm{HSC}^{24}$ and it is possible that loss of this self-renewal division is responsible for the loss of cell numbers of megakaryopoietic HSPC within the bone marrow. Here we show that THPO is required for proliferation of HSPC with megakaryopoietic potential while previous studies showed that it is required for the maturation of megakaryocytes from MkP. Together, this would suggest that THPO plays a dual role in maintaining the megakaryocyte population and that both loss of megakaryocyte-producing HSPC and impairment of MkP maturation lead to the acute reduction of megakaryocytes seen in $\mathrm{KO}$ models. In humans, injection of THPO leads to an autoimmune response against the exogenous protein and even against endogenous THPO. For this reason, the artificial recombi- 
nant romiplostim and the small molecule eltrombopag have been approved as clinical MPL agonists. We showed that treatment of Thpo-KO mice with romiplostim can rescue platelet numbers, indicating that megakarypoietic potential is partially cell-intrinsic. Further investigation may reveal the mechanisms through which THPO/MPL signaling drives expansion of HSPC and its role in selfrenewal division of megakaryopoietic HSPC.

This study addresses the many conflicting reports of the role of THPO in HSPC quiescence/self-renewal division as well as its role in megakaryocyte differentiation. It identifies specific HSPC populations that depend on THPO as well as showing that THPO may not be directly responsible for the loss of quiescence observed in THPO KO models, resolving previous contradictory findings.

\section{Disclosures}

No conflicts of interest to disclose.

\section{Contributions}

$A O^{\prime} N$ conceived and designed the study, collected, analyzed and interpreted data and wrote the manuscript. DC wrote the manuscript; DT and A'QBBAM collected data. AI-N provided study materials and collected data. TS conceived and designed the study, organized financial support and gave final approval of the manuscript.

\section{Acknowledgments}

The authors thank Dr. Md. Zakir Hossain at CSI, NUS for assistance in the design and generation of the Thpo flox mice and Prof. Qingde Wang of University of Pittsburgh Medical Center for kindly providing Alb-Cre mice.

\section{References}

1. Choi JS, Mahadik BP, Harley BA. Engineering the hematopoietic stem cell niche: frontiers in biomaterial science. Biotechnol J. 2015;10(10):1529-1545.

2. Birbrair A, Frenette PS. Niche heterogeneity in the bone marrow. Ann N Y Acad Sci. 2016;1370(1):82-96.

3. de Graaf CA, Metcalf D. Thrombopoietin and hematopoietic stem cells. Cell Cycle. 2011;10(10):1582-1589.

4. Oian H, Buza-Vidas N, Hyland CD, et al. Critical role of thrombopoietin in maintaining adult quiescent hematopoietic stem cells. Cell Stem Cell. 2007;1(6):671-684.

5. de Sauvage FJ, Carver-Moore K, Luoh SM, et al. Physiological regulation of early and late stages of megakaryocytopoiesis by thrombopoietin. J Exp Med. 1996;183(2):651-656.

6. Woolthuis CM, Park CY. Hematopoietic stem/progenitor cell commitment to the megakaryocyte lineage. Blood. 2016;127(10): 1242-1248.

7. Ema H, Takano H, Sudo K, Nakauchi H. In vitro self-renewal division of hematopoietic stem cells. J Exp Med. 2000;192(9):12811288.

8. Nishikii H, Kanazawa Y, Umemoto T, et al. Unipotent megakaryopoietic pathway bridging hematopoietic stem cells and mature megakaryocytes. Stem Cells. 2015;33(7): 2196-2207.

9. Besancenot R, Roos-Weil D, Tonetti C, et al. JAK2 and MPL protein levels determine
TPO-induced megakaryocyte proliferation vs differentiation. Blood. 2014;124(13):21042115.

10. Fleischman AG, Tyner JW. JAK2 V617F down-modulates MPL. Blood. 2012;119(20): 4579-4580.

11. Decker M, Leslie J, Liu Q, Ding L. Hepatic thrombopoietin is required for bone marrow hematopoietic stem cell maintenance. Science. 2018;360(6384):106-110.

12. Pronk CJ, Rossi DJ, Mansson R, et al. Elucidation of the phenotypic, functional, and molecular topography of a myeloerythroid progenitor cell hierarchy. Cell Stem Cell. 2007;1(4):428-442.

13. Oguro H, Ding L, Morrison SJ. SLAM family markers resolve functionally distinct subpopulations of hematopoietic stem cells and multipotent progenitors. Cell Stem Cell. 2013;13(1):102-116.

14. Ema H, Morita Y, Suda T. Heterogeneity and hierarchy of hematopoietic stem cells. Exp Hematol. 2014;42(2):74-82.

15. Yamamoto R, Morita Y, Ooehara J, et al. Clonal analysis unveils self-renewing lineage-restricted progenitors generated directly from hematopoietic stem cells. Cell. 2013;154(5):1112-1126.

16. Luc S, Anderson K, Kharazi S, et al. Downregulation of $\mathrm{Mpl}$ marks the transition to lymphoid-primed multipotent progenitors with gradual loss of granulocyte-monocyte potential. Blood. 2008;111(7):3424-3434.

17. Khoramian Tusi B, Socolovsky M. Highthroughput single-cell fate potential assay of murine hematopoietic progenitors in vitro. Exp Hematol. 2018;60:21-29.

18. Bruns I, Lucas D, Pinho S, et al. Megakaryocytes regulate hematopoietic stem cell quiescence through CXCL4 secretion. Nat Med. 2014;20(11):1315-1320.

19. Nakamura-Ishizu A, Takubo K, Fuijoka M Suda T. Megakaryocytes are essential for HSC quiescence through the production of thrombopoietin. Biochem Biophys Res Commun. 2014;454(2):353-357.

20. Zhao M, Perry JM, Marshall $\mathrm{H}$, et al Megakaryocytes maintain homeostatic quiescence and promote post-injury regeneration of hematopoietic stem cells. Nat Med. 2014;20(11):1321-1326.

21. Greenbaum A, Hsu YM, Day RB, et al CXCL12 in early mesenchymal progenitors is required for haematopoietic stem-cell maintenance. Nature. 2013;495(7440):227-230

22. Veldkamp CT, Peterson FC, Pelzek AJ, Volkman BF. The monomer-dimer equilibrium of stromal cell-derived factor-1 (CXCL 12) is altered by $\mathrm{pH}$, phosphate, sulfate, and heparin. Protein Sci. 2005;14(4):1071-1081.

23. Nakamura-Ishizu A, Matsumura T, Stumpf PS, et al. Thrombopoietin metabolically primes hematopoietic stem cells to megakaryocyte-lineage differentiation. Cell Rep. 2018;25(7):1772-1785.

24. Kovtonyuk IV, Manz MG, Takizawa H Enhanced thrombopoietin but not G-CSF receptor stimulation induces self-renewing hematopoietic stem cell divisions in vivo. Blood. 2016;127(25):3175-3179 